\title{
Novel crystalline carbon-cage structure synthesized from laser-driven shock wave loading of graphite
}

\author{
Sheng-Nian Luo ${ }^{\text {a) }}$ \\ P-24 Plasma Physics, Los Alamos National Laboratory, Los Alamos, New Mexico 87545 \\ Oliver Tschauner \\ High-Pressure Science and Engineering Center and Department of Physics, University of Nevada, \\ Las Vegas, Nevada 89154 \\ Thomas E. Tierney IV and Damian C. Swift \\ P-24 Plasma Physics, Los Alamos National Laboratory, Los Alamos, New Mexico 87545 \\ Steve J. Chipera \\ Earth and Environmental Sciences, Los Alamos National Laboratory, Los Alamos, New Mexico 87545 \\ Paul D. Asimow \\ Division of Geological and Planetary Sciences, California Institute of Technology, Pasadena, \\ California 91125
}

(Received 14 March 2005; accepted 20 May 2005; published online 18 July 2005)

\begin{abstract}
We report a novel crystalline carbon-cage structure synthesized from laser-driven shock wave loading of a graphite-copper mixture to about $14 \pm 2 \mathrm{GPa}$ and $1000 \pm 200 \mathrm{~K}$. Quite unexpectedly, it can be structurally related to an extremely compressed three-dimensional $\mathrm{C}_{60}$ polymer with random displacement of $\mathrm{C}$ atoms around average positions equivalent to those of distorted $\mathrm{C}_{60}$ cages. Thus, the present carbon-cage structure represents a structural crossing point between graphite interlayer bridging and $\mathrm{C}_{60}$ polymerization as the two ways of forming diamond from two-dimensional and molecular carbon. () 2005 American Institute of Physics. [DOI: 10.1063/1.1953562]
\end{abstract}

Understanding the graphite-diamond transition mechanisms including transitory or intermediate phases may open windows for a more efficient synthesis of diamond. The graphite-diamond transition is also interesting by itself as a paradigm of reconstructive transitions involving changes in coordination, bonding and dimensionality, and for its possible relations with other transition routes to diamond from, for instance, $\mathrm{C}_{60}$ polymers. However, the detailed mechanisms still remain unsettled despite considerable experimental and theoretical efforts motivated by the technological and scientific significance.

Carbon is a complicated system ${ }^{1}$ rich in polymorphs due to its ability to form $s p-, s p^{2}$-, and $s p^{3}$-hybridized C-C bonds including molecular phases such as buckyballs and buckytubes, the two-dimensional (2D) graphite, and threedimensional (3D) tetrahedral network of diamond. $\mathrm{C}_{60}$ polymers, ${ }^{2-5}$ carbyne, ${ }^{6}$ and hypothetical cage structures such as carbon zeolites ${ }^{7}$ establish intermediate phases intriguing for their structures and properties. The phase diagram and other high-pressure properties of $\mathrm{C}_{60}$ have been investigated extensively using static techniques. ${ }^{4,8-15}$ The transitions of graphite and buckyballs to diamond involve major reconstruction of bonding and coordination, and thus are loadingrate-dependent processes. Dynamic experiments such as shock waves may be advantageous for revealing the transition mechanisms by metastable recovery of transient structures formed upon ultrafast loading. We have demonstrated

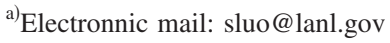

for silica (another paradigm system) that a transient structure intermediate between tetrahedrally and octahedrally coordinated phases forms upon shock wave loading of quartz and coesite. $^{16}$

Diamond was synthesized by conventional shock wave (explosives or gas guns) loading of graphite alone or graphite-copper powder mixture to about $20 \mathrm{GPa}$ and above. ${ }^{17-20}$ Recent explosive-driven shock wave loading of pyrolytic graphite to about $15 \mathrm{GPa}$ yielded several carbon allotropes including carbyne, a diamondlike phase and possibly fullerene. ${ }^{21}$ It is worth noting that $\mathrm{C}_{60}$ fullerene synthesis was pioneered by vaporizing graphite using lasers. ${ }^{22}$ Naturally occurring fullerenes in meteorites and sediments were inferred to have formed during terrestrial impact. ${ }^{23,24}$ Conventional shock wave loading of $\mathrm{C}_{60}$ fullerene yielded amorphous diamond. ${ }^{25}$ The differences in heating and strain rates, porosity, starting materials, pressure-temperature conditions, and inherent kinetics for phase transitions may strongly affect the nature of the structures formed upon loading and upon recovery.

In this work, we utilize laser-induced shock wave loading $^{26,27}$ at higher strain and heating rates and shorter loading durations to probe possible transient structures on the path of graphite-diamond transition. The starting materials were a mixture of graphite and copper powders ( $3: 16$ by mass). The sample pellet, sandwiched between an $\mathrm{Al}$ ablator (12.5 $\mu \mathrm{m}$ thick) and a $\mathrm{LiF}$ window, was subjected to shock wave loading originated in the ablator irradiated by a laser pulse of $\sim 2.4-\mathrm{ns}$ duration, 527-nm wavelength, and 185-J 
energy. (The expansion of the inertially confined vapors and plasmas results in shock waves propagating into the condensed matter and inducing compression and heating.) The laser-spot size was about $5 \mathrm{~mm}$. The peak pressure and temperature were estimated as $14 \pm 2 \mathrm{GPa}$ and $1000 \pm 200 \mathrm{~K}$. Note that the shock wave induced at the laser-irradiated Alablator surface propagated through the remaining part of the ablator and then into the sample, i.e., the sample was not directly subjected to laser irradiation. The remaining ablator and the outermost layer of the sample were pulled off under tension induced by interacting rarefaction waves during release. The recovered sample surface demonstrated fresh powders without an indication of melting or vaporization. Thus our technique is fundamentally different from the laser vaporization of graphite in the fullerene synthesis. ${ }^{22}$

Compared to static high-pressure experiments such as the large-volume press, laser-driven shock wave loading appears to be more prone to nonuniformity of loading, and difficulties in the accurate determination of local pressure and temperature conditions and in situ structure-related measurements such as x-ray diffraction. (Nonetheless, the nonuniformity can be alleviated by spatially smoothing the laser beam with phase plates, and advances have been achieved in time-resolved structure measurements, for example, with transient $\mathrm{x}$-ray diffraction. ${ }^{26,28}$ ) Due to inherent technical limitations, we intended mainly to investigate the structure changes of graphite under high strain- and heating-rate laserdriven shock wave experiments by rapidly quenching structurally modified high-pressure structures to ambient conditions. The issue of structural changes upon release has remained unresolved in general for shock recovery experiments. However, considering the extremely high unloading rate in laser experiments with small samples, it is reasonable to make the assumption that the recovered phase largely represents the structure formed upon shock.

Copper powder was used for thermal quenching and shock impedance modification as in previous diamond synthesis at industrial scale ${ }^{19}$ where there was no firm indication of $\mathrm{Cu}$ being chemically contained in diamond, largely due to the modest shock temperature. Similarly, we do not expect $\mathrm{Cu}$-contained carbon phases in our experiments, although previous experiments using such techniques as laser vaporization, evaporation, or ion implantation ${ }^{29-33}$ have shown that $\mathrm{Cu}$ could be chemically involved with various carbon phases.

For analyses, fractions of material were retrieved from the center of the impacted area. Large grains of $\mathrm{Cu}$ (about $50 \mu \mathrm{m}$ in diameter) were removed mechanically and aggregates of shocked starting material about $100 \mu \mathrm{m}$ in diameter were mounted on top of a borosilicate fiber. X-ray-diffraction images were collected at the 16ID-B undulator beamline at the High Pressure Collaborative Access Team (HPCAT), Sec. 16 of the APS-ANL synchrotron, using a monochromatic beam of $0.3519-\AA$ wavelength and a MAR345 image plate detector with $100 \times 100 \mu \mathrm{m}^{2}$ pixel size placed $350 \mathrm{~mm}$ away from the sample. The $\mathrm{x}$-ray beam was focused to 50 $\times 50 \mu \mathrm{m}^{2}$ on the detector by two Kirkpatrick-Baez mirrors.

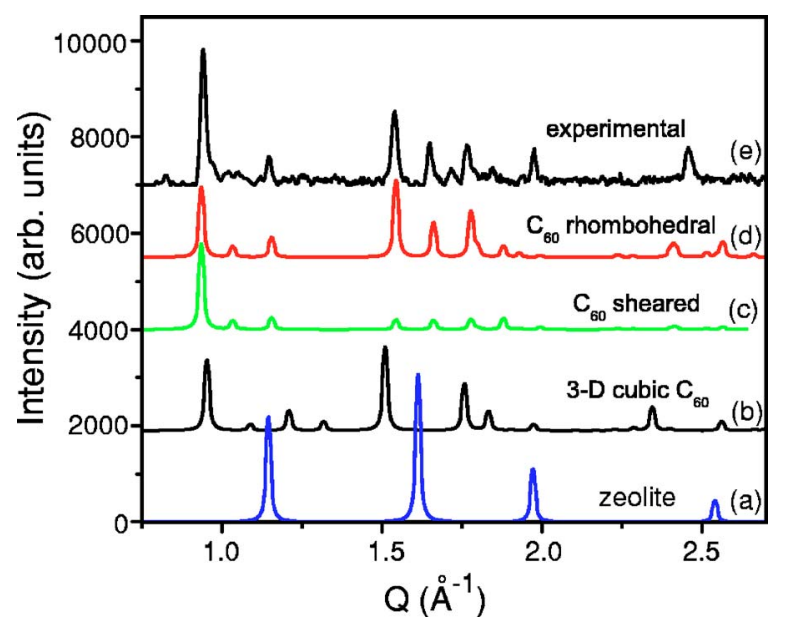

FIG. 1. Comparison of the observed x-ray-diffraction pattern (background subtracted) with the predictions based on typical carbon-cage structures (zeolite and $\mathrm{C}_{60}$-based structures). The patterns from non- $\mathrm{C}_{60}$-based cage structures such as $\mathrm{C}$ zeolite (a) deviate significantly from the observed pattern. Among all $\mathrm{C}_{60}$-based polymers (b)-(d), the rhombohedral one [compressed from the $2 \mathrm{D}$ rhombohedral $\mathrm{C}_{60}$ polymer (Ref. 5)] clearly yields the best match. Thus, the present material is in close structural relation to this rhombohedral $\mathrm{C}_{60}$ polymer.

The sample was rotated along the $\omega$ direction by $\pm 15^{\circ}$ during signal accumulation for $500 \mathrm{~s}$. The diffraction images were integrated and corrected for geometric distortions using FIT2D. ${ }^{34}$

Both amorphous and crystalline phases were identified in the recovered materials. Here we discuss the diffraction data (Figs. 1 and 2) which have indicated the shock synthesis of a novel carbon-cage structure from graphite. The signal was relatively weak due to the small amount of material retrieved. The Bragg peaks observed are clearly from a crystalline phase which is neither graphite nor diamond. Distinct

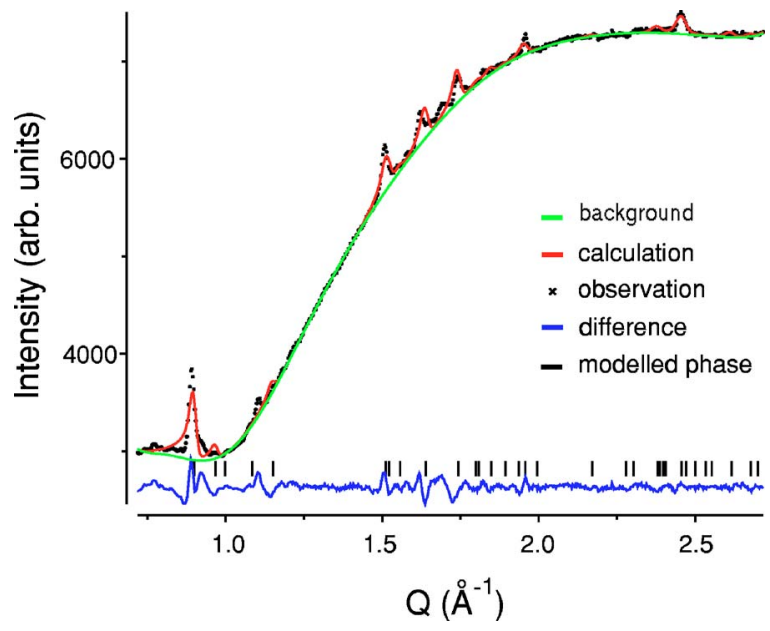

FIG. 2. Comparison of the observed x-ray-diffraction pattern (background included) with the calculated pattern of the refined $\mathrm{C}_{60}$-based structure (monoclinic carbon-cage structure of space group $C 12 / \mathrm{m} 1$ ). The statistical parameters of the Rietveld-refinement (Ref. 41) are $\chi^{2}=8.230, w R_{p}=0.052$, and $R_{p}=0.020$ (without background). There are 637 observations with 41 independent ones. The residual still contains features of other large $\mathrm{C}$ structures (e.g., $Q=0.88$ and $1.1 \AA^{-1}$ ) which cannot be modeled and refined separately and do not belong to the principal phase proposed, as attested by the convergence of the Le Bail and Rietveld refinement to the same measures of goodness of fit (see text). 
Bragg peaks of the crystalline material occur between $Q$ $=0.8$ and $2.6 \AA^{-1}$ (Figs. 1 and 2). (The wave vector $Q$ $=4 \pi \sin \theta / \lambda$ where $\theta$ is the Bragg angle and $\lambda$ is the wavelength.) The extension of Bragg reflections to $Q$ values as low as $0.8 \AA^{-1}$ and the complexity of the pattern between 0.8 and $2.6 \AA^{-1}$ indicate that the present material contains phases with large unit cells $\left(500-1000 \AA^{3}\right)$ and more than just a few atoms in the asymmetric unit. The present material is therefore a candidate for a carbon-cage structure predicted to be transitional between $s p^{2}$-bonded graphite and $s p^{3}$-bonded diamond. ${ }^{7}$ In Fig. 1 we compare the predicted diffraction patterns based on various large carbon-cage structures (both observed and calculated) with the experimental pattern after background subtraction [Fig. 1(e)]. Calculations based on the carbon zeolite and clathrate structures do not match the observed pattern [only the carbon zeolite structure proposed by O'Keeffe $^{7}$ was shown as Fig. 1(a)]. On the other hand, the match in the diffraction patterns is much better between the present material and the cage structures derived from compaction and polymerization of $\mathrm{C}_{60}$. The calculated pattern of the three-dimensionally polymerized $\mathrm{C}_{60}[\text { Fig } 1(\mathrm{~b})]^{2}$ shows a significantly closer similarity to the observed pattern. Hereby we assumed a cubic cell with a cell length of $12 \AA$ (density of $2.77 \mathrm{~g} \mathrm{~cm}^{-3}$ ). An even better match results from a rhombohedral $2 \mathrm{D}$ polymer ${ }^{5}$ artificially compacted to a cell volume of about $1080 \AA^{3}$ [density of $3.3 \mathrm{~g} \mathrm{~cm}^{-3}$; Fig. 1(d)]. In addition, we have shown a $\mathrm{C}_{60}$-based phase where the $\mathrm{C}_{60}$ cages are sheared in one plane [the (111) plane of a pseudorhombohedral triclinic cell; Fig. 1(c)]. The comparisons strongly indicate that the present material is structurally close to a compacted $\mathrm{C}_{60}$ polymer.

We note that the limited angular range (low-angle regime) and low resolution resulting from the high x-ray energy and the use of an image plate detector do not allow us to constrain individual atomic positions solely from the diffraction data. However, the low-angle peaks have proved to be highly distinctive and diagnostic for the various $\mathrm{C}_{60}$ polymers. ${ }^{15,35-38}$ This is due to the fact that these phases are composed of identical large subunits (the $\mathrm{C}_{60}$ cages) which are arranged relative to each other and connected in different ways. Thus, the angular range shown in Fig. 1 is relevant for determining the principal structural type of the present large cage structure, although our data are not sufficient to reliably determine individual atomic positions in a unit cell for such a large structure. In fact, the match between the diffraction patterns from the compacted rhombohedral polymer [Fig. 1(d)] and the present material [Fig. 1(e)] is very close, while even moderate changes in the structure [Figs. 1(b) and 1(c)] have induced marked discrepancies, suggesting a close structural relation between the compacted rhombohedral $\mathrm{C}_{60}$ polymer and the present phase. The compacted rhombohedral polymer is therefore a good structure model for the present material. Although the known rhombohedral polymers of $\mathrm{C}_{60}$ are $2 \mathrm{D},{ }^{5,35}$ the much higher compaction in the present case induces further intercage bonding in the third dimension. The material is therefore a $3 \mathrm{D}$ polymer with structural anisotropy, in contrast with the 3D crystalline polymer reported from static compression experiments on $\mathrm{C}_{60}{ }^{9,39,40}$ While the present phase is derived from the com- pacted rhombohedral $\mathrm{C}_{60}$, its symmetry may be lower than the rhombohedral structure while the close match of the model with the observed pattern suggests that a symmetry reduction is constrained by group-subgroup relations.

We examined this possibility by refining the cell of our model structure while releasing all symmetry constraints on the compacted rhombohedral structure but keeping the atoms at fixed positions and optimized the cell parameters by the Rietveld refinement, ${ }^{41,42}$ in order to anchor the strong reflections by the observed peak intensities, thus avoiding unphysical drift of cell parameters. In a second step we also refined the structure factors. Surprisingly, the structure-factor refinement converged without large shifts in atomic coordinates. As mentioned above, the individual atomic positions in such a large structure with a large asymmetric unit are not well constrained by the given data. However, the convergence of refinement confirms the stability of the model structure relative to the observed pattern. We continued iterating alternately the refinement of the cell parameters and structure factors. In the final step we searched for space groups consistent with the refined cell. This procedure resulted in a pseudohexagonal, monoclinic cell of space group $C 12 / \mathrm{m} 1$. We checked and confirmed the stability of the monoclinic cell by conducting extra cycles of Rietveld refinement, and the result is shown in Fig. 2. We also performed the Le Bail extraction $^{43}$ which yielded equally good statistical measures of goodness $\left(w R_{p} \text { and } \chi^{2}\right)^{41}$ as the Rietveld refinement while converging to the same cell, rather than diverging or drifting to different cells, as expected for the cases of worse match of calculated structure factors and those extracted by the Le Bail method.

The cell parameters (Table I) are $a=14.497(9) \AA, b$ $=8.067(13) \AA, c=8.359(8) \AA$, and $\beta=131.11(5)^{\circ}$ for an optimized space group $C 12 / \mathrm{m} 1$, which is a translationsgleiche subgroup of $R \overline{3} m$, the space group of the rhombohedral 2D $\mathrm{C}_{60}$ polymer described by Chen et al. ${ }^{5}$ The cell volume is $736.5(6) \AA^{3}$ and the calculated density $3.251 \mathrm{~g} \mathrm{~cm}^{-3}$. The angular distortion is $1.7^{\circ}$. The atomic positions in the unit cell of the optimized structure (Fig. 3) are listed in Table I. In static experiments under similar pressure and temperature conditions, the disordered networks of density between 3 and $3.4 \mathrm{~g} \mathrm{~cm}^{-3}$ have been reported. ${ }^{3,9,39,44}$ Thus, the density of the present new phase is well within the expected range. However, the present phase is crystalline, while these static experiments reported amorphous phases only.

The cell dimensions of the refined model imply a compression of the underlying buckyball units to the internal diameters of 5.3 and $6.4 \AA$. For comparison, in the $2 \mathrm{D} \mathrm{C}_{60}$ polymers these diameters are ${ }^{5,35} 6.54$ and $7.59 \AA$, and in the 3D random-bonded fullerites they are $7.2 \AA$ on average. ${ }^{2}$ Hence, if the proposed structure model is valid, the $\mathrm{C}_{60}$ cages have to be deformed (corrugated) to a large extent in order to relax the C-C bond distances to 1.4-1.6 A. Such corrugation of buckyballs has already been reported for $3 \mathrm{D} \mathrm{C}_{60}$ polymers synthesized under static compression and heating. ${ }^{2,3,39}$ This raises the question if a cage structure derived from corrugation of $\mathrm{C}_{60}$ gives a better representation of the present phase than the modeled "overcompressed" $\mathrm{C}_{60}$ polymer (Table I and Fig. 3) which represents the average atomic positions in 
TABLE I. Fractional coordinates $(x, y$, and $z)$ of the atoms for the refined $\mathrm{C}_{60}$-based carbon-cage structure model (space group $C 12 / \mathrm{m} 1$ ). The unit-cell parameters are $a=14.497(9) \AA, b=8.067(13) \AA, c=8.359(8) \AA$, and $\beta$ $=131.11(5)^{\circ}$. The cell volume is $736.5(6) \AA^{3}$ and the calculated density $3.251(26) \mathrm{g} \mathrm{cm}^{-3}$. The unit cell contains 120 atoms. The thermal displacement factors were set to $0.025 \AA^{2}$. The relative errors in the fractional coordinates are about $5 \%-8 \%$. Wyckoff denotes atomic multiplicity and symmetry in Wyckoff notation. Note that the atomic positions were not obtained by independent refinement which is not allowed by the limited diffraction data, instead, they represent the average atomic coordinates in the optimized structure starting from the rhombohedral $\mathrm{C}_{60}$ structure.

\begin{tabular}{lcccc}
\hline \hline Atom & Wyckoff & $x$ & $y$ & $z$ \\
\hline $\mathrm{C} 1$ & $8 \mathrm{j}$ & 0.2147 & 0.1270 & 0.4654 \\
$\mathrm{C} 2$ & $8 \mathrm{j}$ & 0.3108 & 0.7766 & 0.6549 \\
$\mathrm{C} 3$ & $8 \mathrm{j}$ & 0.4401 & 0.0900 & 0.9159 \\
$\mathrm{C} 4$ & $4 \mathrm{i}$ & 0.7635 & 0.0000 & 0.5807 \\
$\mathrm{C} 5$ & $8 \mathrm{j}$ & 0.0941 & 0.6649 & 0.2473 \\
$\mathrm{C} 6$ & $8 \mathrm{j}$ & 0.1345 & 0.0709 & 0.4538 \\
$\mathrm{C} 7$ & $8 \mathrm{j}$ & 0.3000 & 0.7586 & 0.7863 \\
$\mathrm{C} 8$ & $8 \mathrm{j}$ & 0.3729 & 0.1637 & 0.9292 \\
$\mathrm{C} 9$ & $4 \mathrm{i}$ & 0.7453 & 0.0000 & 0.7028 \\
$\mathrm{C} 10$ & $8 \mathrm{j}$ & 0.0257 & 0.7182 & 0.2669 \\
$\mathrm{C} 11$ & $8 \mathrm{j}$ & 0.9096 & 0.2828 & 0.1001 \\
$\mathrm{C} 12$ & $8 \mathrm{j}$ & 0.2586 & 0.3723 & 0.7990 \\
$\mathrm{C} 13$ & $8 \mathrm{j}$ & 0.0500 & 0.8482 & 0.3795 \\
$\mathrm{C} 14$ & $8 \mathrm{j}$ & 0.9542 & 0.0816 & 0.2870 \\
$\mathrm{C} 15$ & $8 \mathrm{j}$ & 0.2925 & 0.5800 & 0.9659 \\
$\mathrm{C} 16$ & $8 \mathrm{j}$ & 0.3665 & 0.3406 & 0.1137 \\
\hline \hline
\end{tabular}

randomly corrugated $\mathrm{C}_{60}$ cages. We modeled further such cagestructures derived from the buckyballs with nonrandom corrugation while keeping the cage centers the same as in the monoclinic phase. However, the match between the calculated and observed intensities is certainly worse for such models than for the monoclinic phase. Thus the present phase can be better described by the monoclinic phase composed of polymerized $\mathrm{C}_{60}$-like shells with strong but random corrugation, i.e., the average atomic positions are those of an extremely compressed $\mathrm{C}_{60}$ polymer (Fig. 3).

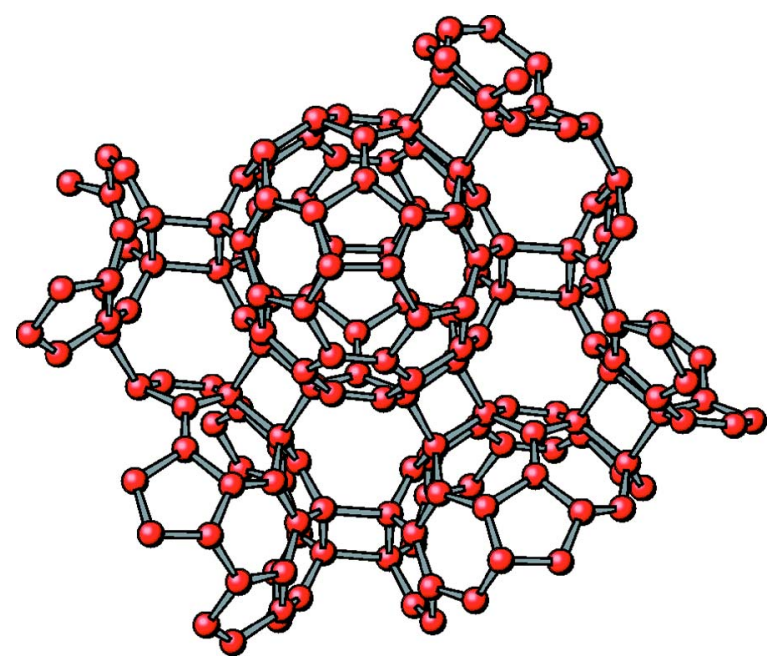

FIG. 3. Structure representation of the modeled carbon-cage structure: the pseudorhombohedral, monoclinic cell with space group $C 12 / \mathrm{m} 1$. This structure represents the average positions of $\mathrm{C}$ atoms (Table $\mathrm{I}$ ) forming two highly compressed, elliptically distorted, and connected $\mathrm{C}_{60}$ cages.
The present crystalline phase therefore represents a structural crossing point of the graphite-diamond and $\mathrm{C}_{60}$-diamond transformation paths, and it is metastable in nature at ambient conditions as evidenced by the fact that it became amorphous in about one month after its synthesis. While it was formed by shock loading of graphite, it is structurally equivalent to an overcompressed $\mathrm{C}_{60}$ polymer with intrinsic but random corrugation of the $\mathrm{C}_{60}$ cages compensating for this large degree of compression. We emphasize that the level of network connectivity and cage deformation is appreciable in comparison to regular buckyballs. Therefore, the present material should not be addressed as a buckyball phase: it is simply on the structural extension of the $\mathrm{C}_{60}$ polymerization and compaction path as well as on the physical transformation path between $s p^{2}$-bonded layered graphite and $s p^{3}$-bonded $3 \mathrm{D}$ tetrahedral diamondlike network. Different fullerene-related structures $2-5,9,15,21-24,35-40$ including isolated molecules, nanoclusters of buckyballs with various sizes and constitutions, and 1D to 3D polymers with differing structural anisotropy and crystallinity have been formed in such loading conditions as laser vaporization, static high-pressure experiments, natural occurrence, and conventional and laser-driven shock wave loading. The present phase represents the sterical limit of $\mathrm{C}_{60}$-based structures: the high degree of compaction is compensated by intrinsic disorder expressed in random corrugation of the cages. This is further illustrated by the rapid amorphization of the present phase and the fact that static hot compression experiments $9,39,40$ yielded only amorphous material with mixed $s p^{2}-s p^{3}$ bond hybridization at the pressure and temperature comparable to our experiment. Interestingly, conventional shock wave loading of $\mathrm{C}_{60}$ yielded amorphous diamondlike material $^{25}$ at lower loading and unloading rates. The ultrafast nature of loading to elevated pressure and temperature in our experiment and the loss of the crystallinity of the present phase at ambient conditions over a short period (compared to diamond) indicate that the present structure indeed represents an intermediate structural stage in the graphite-diamond transition. The close structural relationship of the present phase to $\mathrm{C}_{60}$-based polymers is consistent with the fact that the graphite-diamond and $\mathrm{C}_{60}$-diamond transition routes must converge. The present phase as the structural crossing point is thus important for eventual revelation of the detailed mechanisms for the transitions to diamond.

In summary, laser-induced shock wave loading of a graphite and copper mixture to about $14 \pm 2 \mathrm{GPa}$ and $1000 \pm 200 \mathrm{~K}$ yielded a novel 3D crystalline carbon-cage structure occupying a transitional state intermediate between $\mathrm{C}_{60}$ polymerization and graphite interlayer bridging. The present phase has an ambient density of $3.25 \mathrm{~g} \mathrm{~cm}^{-3}$. Using the $\mathrm{x}$-ray-diffraction data, we modeled the structure of this new phase as a framework of carbon-cages equivalent to three-dimensionally polymerized and randomly corrugated $\mathrm{C}_{60}$ units. Compared to known $3 \mathrm{D}$ polymers of $\mathrm{C}_{60}$, this structure contains a larger number of cage-connecting bonds in two dimensions than in the third, thus reflecting the initial 2D nature of the starting material graphite. Current results have demonstrated that laser-driven shock waves, characterized by ultrashort time scales, supply unique conditions for 
synthesis of novel carbon phases not accessible by static experiments, and may help to elucidate the mechanism for graphite-diamond and $\mathrm{C}_{60}$-diamond transformations.

One of the authors (S.N.L.) was sponsored by a Director's Postdoctoral Fellowship at LANL. We are grateful for the invaluable support from the Trident laser facility/staff and the Inertial Confinement Fusion program at LANL. Another author (O.T.) acknowledges support by the NNSA Cooperative Agreement No. DE-FC88-01NV14049. Use of the HPCAT facility at APS was supported by DOE-BES, DOENNSA, NSF, DOD-TACOM, and the W. M. Keck Foundation. APS at Argonne National Laboratory is a national synchrotron-radiation light source research facility funded by the U.S. Department of Energy under Contract No. W-31109-ENG-38. This work was performed in part under the auspices of U.S. Department of Energy under Contract No. W-7405-ENG-36.

${ }^{1}$ E. D. Miller and D. C. Nesting, Chem. Mater. 9, 18 (1997).

${ }^{2}$ L. Marques, M. Mezouar, J. L. Hodeau, M. Nunez-Regueiro, N. R. Serebryanaya, V. A. Ivdenko, V. D. Blank, and G. A. Dubitsky, Science 283 1720 (1999).

${ }^{3}$ V. D. Blank, B. A. Kulnitskiy, Y. V. Tatyanin, and O. M. Zhigalina, in Electron Microscopy and Analysis 1997, edited by J. M. Rodenburg (Institute of Physics Publishing, Bristol, UK, 1997), p. 536.

${ }^{4}$ B. Sundqvist, Adv. Phys. 48, 1 (1999).

${ }^{5}$ X. Chen, S. Yamanaka, K. Sako, Y. Inoue, and M. Yasukawa, Chem. Phys. Lett. 356, 291 (2002).

${ }^{6}$ A. G. Whittacker, Science 200, 763 (1978).

${ }^{7}$ M. O'Keeffe, Nature (London) 352, 674 (1991).

${ }^{8}$ B. Sunqvist, J. Phys.: Condens. Matter 14, 10449 (2002).

${ }^{9}$ V. V. Brazhkin, A. G. Lyapin, S. V. Popova et al., JETP Lett. 76, 681 (2002).

${ }^{10}$ M. E. Kozlov and K. Yakushi, J. Phys.: Condens. Matter 7, L209 (1995)

${ }^{11}$ A. M. Rao, P. C. Eklund, U. D. Venkateswaran et al., Appl. Phys. A: Mater. Sci. Process. 64, 231 (1997).

${ }^{12}$ V. A. Davydov, L. S. Kashevarova, A. V. Rakhmanina, V. M. Senyavin, R. Ceolin, H. Szwarc, H. Allouchi, and V. Agafonov, Phys. Rev. B 61, 11936 (2000).

${ }^{13}$ Y. Li, J. H. Rhee, D. Singh, and S. C. Sharma, Phys. Rev. B 68, 024106 (2003).

${ }^{14}$ S. Berber, E. Osawa, and D. Tománek, Phys. Rev. B 70, 085417 (2004).

${ }^{15}$ R. Moret, P. Launois, T. Wagberg, B. Sundqvist, V. Agafonov, V. A. Davydov, and A. V. Rakhmanina, Eur. Phys. J. B 37, 25 (2004).

${ }^{16}$ S. N. Luo, O. Tschauner, P. D. Asimow, and T. J. Ahrens, Am. Mineral. 89, 455 (2004).
${ }^{17}$ P. S. DeCarli and J. C. Jamieson, Science 133, 1821 (1961).

${ }^{18}$ R. G. McQueen and S. P. Marsh, in Behavior of Dense Media Under High Dynamic Pressure (Gordon and Breach, New York, 1968).

${ }^{19}$ G. R. Cowan, B. W. Dunnington, and A. H. Holtzman, U.S. Patent No. 3,401,019 (1968).

${ }^{20}$ H. Hirai, S. Kukino, and K. Kondo, J. Appl. Phys. 78, 3052 (1995).

${ }^{21}$ K. Yamada, Y. Tanabe, and A. B. Sawaoka, Philos. Mag. A 80, 1811 (2000).

${ }^{22}$ H. W. Kroto, J. R. Heath, S. C. O’Brien, R. F. Curl, and R. E. Smalley, Nature (London) 318, 162 (1985).

${ }^{23}$ L. Becker, R. J. Poreda, and T. E. Bunch, Proc. Natl. Acad. Sci. U.S.A. 97, 2979 (2000).

${ }^{24}$ L. Becker, R. J. Poreda, A. G. Hunt, T. E. Bunch, and M. Rampino, Science 291, 1530 (2001).

${ }^{25}$ H. Hirai, Y. Tabira, K. Kondo, and N. Ishizawa, Phys. Rev. B 52, 6162 (1995).

${ }^{26}$ S. N. Luo, D. C. Swift, T. E. Tierney et al., High Press. Res. 24, 409 (2004).

${ }^{27}$ D. C. Swift, T. E. Tierney, R. A. Kopp, and J. T. Gammel, Phys. Rev. E 69, 036406 (2004)

${ }^{28}$ D. C. Swift, G. J. Ackland, A. Hauer, and G. A. Kyrala, Phys. Rev. B 64, 214107 (2001).

${ }^{29}$ H. Abe, S. Yamamoto, A. Miyashita, and K. E. Sickafus, J. Appl. Phys. 90, 3353 (2001).

${ }^{30}$ J. Jiao and S. Seraphin, J. Appl. Phys. 83, 2442 (1998).

${ }^{31}$ A. V. Prikhod'ko and O. I. Kon'kov, Semiconductors 35, 659 (2001).

${ }^{32}$ V. Z. Mordkovich and Y. Takeuchi, Chem. Phys. Lett. 355, 133 (2002).

${ }^{33}$ X. Li, Y. J. Tang, H. W. Zhao, W. S. Zhan, H. Wang, and J. G. Hou, Appl. Phys. Lett. 77, 984 (2000).

${ }^{34}$ A. P. Hammersley, S. O. Svensson, M. Hanfland, A. N. Fitch, and D. Hausermann, High Press. Res. 14, 235 (1996).

${ }^{35}$ M. Nunez-Regueiro, L. Marques, J. L. Hodeau, O. Bethoux, and M. Perroux, Phys. Rev. Lett. 74, 278 (1995).

${ }^{36}$ V. A. Davydov, L. S. Kashevarova, A. V. Rakhmanina et al., Phys. Rev. B 58, 14786 (1998).

${ }^{37}$ N. R. Serebryanaya and L. A. Chernozatonskii, Solid State Commun. 114, 537 (2000).

${ }^{38}$ N. R. Serebryanaya, V. D. Blank, V. A. Ivdenko, and L. A. Chernozatonskii, Solid State Commun. 118, 183 (2001).

${ }^{39}$ V. D. Blank, S. G. Buga, G. A. Dubitsky, N. R. Serebryanaya, M. Y. Popov, and B. Sundqvist, Carbon 36, 319 (1998).

${ }^{40}$ M. Mezouar, L. Marques, J. L. Hodeau, V. Piscedda, and M. NunezRegueiro, Phys. Rev. B 68, 193414 (2003).

${ }^{41}$ A. C. Larson and R. B. von Dreele, General Structure Analysis Software (GSAS), LANL Report No. LAUR 86-748, Los Alamos National Laboratory, NM, 1995.

${ }^{42}$ B. H. Toby, J. Appl. Crystallogr. 34, 210 (2001).

${ }^{43}$ A. Le Bail, H. Duroy, and J. L. Fourquet, Mater. Res. Bull. 23, 447 (1988).

${ }^{44}$ L. Marques, J. L. Hodeau, M. N. Nunez-Regueiro, and M. Perroux, Phys. Rev. B 54, R12633 (1996) 